\title{
THE ORDER OF PERIODIC ELEMENTS OF TEICHMÜLLER MODULAR GROUPS
}

\author{
EGE FUJIKAWA
}

\begin{abstract}
We consider a quasiconformal automorphism of a Riemann surface, which fixes the homotopy class of a simple closed geodesic. Under certain conditions on the injectivity radius of the surface and bounds on the dilatation of the map, the automorphism induces a periodic element of the Teichmüller modular group. We may also estimate the order of the period.
\end{abstract}

\section{INTRODUCTION}

Let $R$ be an arbitrary Riemann surface with possibly infinitely generated fundamental group. An element $\chi$ of the Teichmüller modular group $\operatorname{Mod}(R)$ is induced by a quasiconformal automorphism $f$ of $R$. We would like to determine when the order of $\chi$ is finite. When $f$ is a conformal automorphism of $R$, then the element $\chi$ of $\operatorname{Mod}(R)$ induced by $f$ fixes the base point of the Teichmüller space $T(R)$. In [3], we proved that, for a Riemann surface $R$ with non-abelian fundamental group, a conformal automorphism $f$ of $R$ has finite order if and only if $f$ fixes either a simple closed geodesic, a puncture or a point on $R$. In each case, we obtained a concrete estimate for the order of $f$ in terms of the injectivity radius on $R$. One of our results is the following. For the definition of the upper bound condition, see the next section.

Theorem 1.1 ([3], [4]). Let $R$ be a hyperbolic Riemann surface with non-abelian fundamental group. Suppose that $R$ satisfies the upper bound condition for a constant $M>0$ and a connected component $R_{M}^{*}$ of $R_{M}$. Let $f$ be a conformal automorphism of $R$ such that $f(c)=c$ for a simple closed geodesic $c$ on $R$ with $c \subset R_{M}^{*}$ and $l(c)=l>0$. Then the order $n$ of $f$ satisfies

$$
n<\left(e^{M}-1\right) \cosh (l / 2) .
$$

The purpose of this paper is to extend Theorem 1.1 to a quasiconformal automorphism $f$. One of the difficulties that arise is that the element $\chi \in \operatorname{Mod}(R)$ induced by $f$ need not have a fixed point on

1991 Mathematics Subject Classification. Primary 30F60; Secondary 30C62. 
$T(R)$. However, we will show that if the maximal dilatation of $f$ is smaller than some constant, then $\chi$ is periodic.

The author would like to express her gratitude to Professor Katsuhiko Matsuzaki for his valuable suggestions.

\section{Statement of Theorem}

Let $\mathbb{H}$ be the upper half-plane equipped with the hyperbolic metric $|d z| / \operatorname{Im} z$. Throughout this paper, we assume that a Riemann surface $R$ is hyperbolic. Namely, it is represented as $\mathbb{H} / \Gamma$ for some torsionfree Fuchsian group $\Gamma$ acting on $\mathbb{H}$. Furthermore, we also assume that $R$ has a non-abelian fundamental group. The hyperbolic distance on $\mathbb{H}$ is denoted by $d$, and the hyperbolic length of a curve $c$ on $R$ by $l(c)$. For the axis $L$ of a hyperbolic element of the Fuchsian group $\Gamma$, we denote by $\pi_{\Gamma}(L)$ the projection of $L$ to $\mathbb{H} / \Gamma$. When there is no fear of confusion, we denote this simply by $\pi(L)$. Also, for a quasiconformal automorphism $\tilde{f}$ of $\mathbb{H}$, we denote by $\tilde{f}(L)_{*}$ the geodesic having the same end points as those of $\tilde{f}(L)$.

We recall the definition of Teichmüller spaces and Teichmüller modular groups. Fix a Riemann surface $R$. We say that two quasiconformal maps $f_{1}$ and $f_{2}$ on $R$ are equivalent if $f_{2} \circ f_{1}^{-1}$ is homotopic to a conformal map of $f_{1}(R)$ onto $f_{2}(R)$. The reduced Teichmüller space $T(R)$ with the base Riemann surface $R$ is the set of all equivalence classes $[f]$ of quasiconformal maps $f$ on $R$. The Teichmüller distance $d_{T}$ on $T(R)$ is defined by $d_{T}\left(\left[f_{1}\right],\left[f_{2}\right]\right)=\log K(g)$, where $g$ is an extremal quasiconformal map in the sense that its maximal dilatation $K(g)$ is minimal in the homotopy class of $f_{2} \circ f_{1}^{-1}$. This is a complete metric on $T(R)$. The reduced Teichmüller modular group $\operatorname{Mod}(R)$ of $R$ is a group of the homotopy classes $[h]$ of quasiconformal automorphisms $h$ of $R$. Each element $[h]$ of $\operatorname{Mod}(R)$ induces an automorphism of $T(R)$ by $[f] \mapsto\left[f \circ h^{-1}\right]$, which is an isometry with respect to $d_{T}$.

We now make a couple of definitions given in terms of the hyperbolic geometry of Riemann surfaces.

Definition. For a constant $M>0$, we define $R_{M}$ to be the set of points $p \in R$ for which there exists a non-trivial simple closed curve $c_{p}$ passing through $p$ with $l\left(c_{p}\right)<M$. The set $R_{\epsilon}$ is called the $\epsilon$-thin part of $R$ if $\epsilon>0$ is smaller than the Margulis constant. Furthermore, a connected component of the $\epsilon$-thin part corresponding to a puncture is called the cusp neighborhood.

REMARK. The injectivity radius at a point $p \in R$ is the supremum of radii of embedded hyperbolic discs centered at $p$. Note that $R_{M}$ 
coincides with the set of those points having the injectivity radius less than $M / 2$.

DEFinition. We say that $R$ satisfies the lower bound condition if there exists a constant $\epsilon>0$ such that $\epsilon$-thin part of $R$ consists of only cusp neighborhoods or neighborhoods of geodesics which are homotopic to boundary components. We also say that $R$ satisfies the upper bound condition if there exist a constant $M>0$ and a connected component $R_{M}^{*}$ of $R_{M}$ such that the homomorphism of $\pi_{1}\left(R_{M}^{*}\right)$ to $\pi_{1}(R)$ induced by the inclusion map of $R_{M}^{*}$ into $R$ is surjective.

REMARK. The lower and upper bound conditions are quasiconformally invariant notions (see [5, Lemma 8]).

We shall obtain a range of maximal dilatations of quasiconformal automorphisms $f$ inducing periodic elements $\chi \in \operatorname{Mod}(R)$. Moreover, we get a concrete estimate for the order of $\chi$.

Theorem 2.1. Let $R$ be a Riemann surface satisfying the lower bound condition for a constant $\epsilon>0$ as well as the upper bound condition for a constant $M>0$ and a connected component $R_{M}^{*}$ of $R_{M}$. For a given constant $l>0$, there exists a constant $K_{0}=K_{0}(\epsilon, M, l)>1$ depending only on $\epsilon, M$ and $l$ that satisfies the following: Let $f$ be a quasiconformal automorphism of $R$ such that $f(c)$ is homotopic to $c$ for a simple closed geodesic $c$ on $R$ with $c \subset R_{M}^{*}$ and $l(c)=l$. Suppose $K(f)<K_{0}$. Then there exists a positive integer $n \leq N_{0}$ such that $f^{n}$ is homotopic to the identity. Here

$$
\begin{gathered}
N_{0}=N_{0}(M, l)=-\frac{l}{\log (\tanh (D+13.5))}, \\
D=D(M, l)= \begin{cases}2 \operatorname{arccosh}\left(\frac{\sinh (M / 2)}{\sinh (l / 2)}\right)+M & \text { if } l \leq M, \\
M & \text { if } l \geq M .\end{cases}
\end{gathered}
$$

In particular, when $K(f)=1$, we have the following:

Theorem 2.2. Let $R$ be a Riemann surface satisfying the upper bound condition for a constant $M>0$ and a connected component $R_{M}^{*}$ of $R_{M}$ as well as the lower bound condition. Let $f$ be a conformal automorphism of $R$ such that $f(c)=c$ for a simple closed geodesic $c$ on $R$ with $c \subset R_{M}^{*}$ and $l(c)=l>0$. Then the order $n$ of $f$ satisfies

$$
n \leq-\frac{l}{\log (\tanh (D / 2))},
$$

where $D=D(M, l)$ is the same constant as in Theorem 2.1. 
Note that for $M \geq \operatorname{arcsinh}(2 / \sqrt{3})=0.98 \cdots$ and every $l>0$, we have

$$
-\frac{l}{\log (\tanh (M / 2))}<\left(e^{M}-1\right) \cosh (l / 2) .
$$

Here the constant $\operatorname{arcsinh}(2 / \sqrt{3})$ is the smallest possible value of $M$ for which $R$ satisfies the upper bound condition (see [6]). Hence when $l \geq M$, the upper bound of the order of $f$ obtained in Theorem 2.2 is smaller than that in Theorem 1.1. However, when $l<M$, the estimate in Theorem 1.1 is still better than that in Theorem 2.2 for all sufficiently small $l$. In fact, $\left(e^{M}-1\right) \cosh (l / 2)$ converges to $e^{M}-1$ as $l \rightarrow 0$, while $-l /(\log (\tanh (D / 2)))$ diverges to $+\infty$.

In connection with Theorems 2.1 and 2.2 , we would like to mention the result about the discreteness of the orbit of a certain subgroup of the Teichmüller modular group.

Proposition 2.3 ([5]). Let $R$ be a Riemann surface satisfying the lower and upper bound conditions. For a simple closed geodesic $c$ on $R$, let $G$ be a subgroup of $\operatorname{Mod}(R)$ such that $g(c)$ is homotopic to $c$ for every $[g] \in G$. Then for every point $p \in T(R)$, the orbit $G(p)$ of $p$ is a discrete subset in $T(R)$. Furthermore, for any point $p \in T(R)$, there exist only finitely many elements $[g]$ in $G$ that fix $p$.

\section{Proof of Theorems}

For a proof of these theorems, we first prove some properties on the hyperbolic geometry of Riemann surfaces.

Proposition 3.1. Let $R=\mathbb{H} / \Gamma$ be a Riemann surface satisfying the upper bound condition for a constant $M>0$ and a connected component $R_{M}^{*}$ of $R_{M}$. Suppose that $L$ is the axis of a hyperbolic element of $\Gamma$ such that the projection $\pi(L)$ is a simple closed geodesic $c$ on $R$ with $c \subset R_{M}^{*}$ and $l(c)=l>0$. Then there exists an axis $L^{\prime}$ of a hyperbolic element of $\Gamma$ such that $L \cap L^{\prime}=\emptyset, d\left(L, L^{\prime}\right) \leq D$ and $\pi\left(L^{\prime}\right)=\pi(L)$. Here $D=D(M, l)$ is the same constant as in Theorem 2.1.

Proof. First we assume that $l>M$. Since $c \subset R_{M}^{*}$, there exists a non-trivial simple closed curve $\alpha$ passing through $p \in c$ with $l(\alpha)<M$. It follows from the assumption $l>M$ that $\alpha$ is not homotopic to $c$, which implies that there exists an axis $L^{\prime}(\neq L)$ such that $\pi\left(L^{\prime}\right)=c$ and $d\left(L, L^{\prime}\right)<M$. 
Next we assume that $l \leq M$. We further assume that there exists an annular neighborhood $A(c)$ of $c$ with width $\omega(c)$, where

$$
\omega(c)=\operatorname{arccosh}\left(\frac{\sinh (M / 2)}{\sinh (l / 2)}\right) \text {. }
$$

Then, for any $q \in \partial A(c)$, the boundary of $A(c)$, the shortest simple closed curve $\gamma$ passing through $q$ and homotopic to $c$ has length $M$.

Indeed, we may assume that $L=\{i y \mid y>0\}$, and $\tilde{q}=e^{i \theta}$ and $\tilde{q}^{\prime}=e^{l+i \theta}$ are lifts of $q$ to $\mathbb{H}$. Then, by the equality (7.20.3) in [2], we have

$$
\frac{1}{\sin \theta}=\frac{1}{\cos (\pi / 2-\theta)}=\cosh d(\tilde{q}, L)=\cosh \omega(c)=\frac{\sinh (M / 2)}{\sinh (l / 2)} .
$$

Thus, by Theorem 7.2.1 in [2], we see that

$$
\sinh \frac{1}{2} d\left(\tilde{q}, \tilde{q}^{\prime}\right)=\frac{\left|\tilde{q}-\tilde{q}^{\prime}\right|}{2\left(\operatorname{Im} \tilde{q} \operatorname{Im} \tilde{q}^{\prime}\right)^{1 / 2}}=\frac{e^{l}-1}{2 e^{l / 2} \sin \theta}=\frac{\sinh (l / 2)}{\sin \theta}=\sinh \frac{M}{2},
$$

which implies that $l(\gamma)=d\left(\tilde{q}, \tilde{q}^{\prime}\right)=M$.

We can take a point $q_{0} \in \partial A(c)$ such that $q_{0} \in R_{M}^{*}$. Indeed, otherwise, $\partial A(c) \cap R_{M}^{*}=\emptyset$. Since $c \subset R_{M}^{*}$, this means that $R_{M}^{*}$ is an annular neighborhood of $c$, contradicting the upper bound condition.

By the definition of $R_{M}$, there exists a non-trivial simple closed curve $\beta$ passing through $q_{0}$ with $l(\beta)<M$. By the consideration above, we see that the curve $\beta$ is not homotopic to $c$. Hence there exists an axis $L^{\prime}(\neq L)$ such that $\pi\left(L^{\prime}\right)=c$ and $d\left(L, L^{\prime}\right)<2 \omega(c)+M$.

Finally, we assume that $l \leq M$ and that the width of the maximal annular neighborhood $A(c)$ of $c$ is less than $\omega(c)$. Then there exists an axis $L^{\prime}(\neq L)$ such that $\pi\left(L^{\prime}\right)=c$ and $d\left(L, L^{\prime}\right)<2 \omega(c)$.

We now estimate the number of axes satisfying Proposition 3.1.

Definition. For an element $\gamma$ of a Fuchsian group, we say that two axes $L_{1}$ and $L_{2}$ are $\gamma$-equivalent if $\gamma^{n}\left(L_{1}\right)=L_{2}$ for some $n \in \mathbb{Z}$.

Proposition 3.2. Let $R=\mathbb{H} / \Gamma$ be a Riemann surface and $D_{0}>$ 0 a constant. Furthermore, let $L$ be the axis of a hyperbolic element $\gamma \in \Gamma$ such that the projection $\pi(L)$ is a simple closed geodesic $c$ on $R$ with $l(c)=l>0$. Let $S$ be the set of axes $L^{\prime}$ of hyperbolic elements of $\Gamma$ satisfying the following: (i) $L \cap L^{\prime}=\emptyset$, (ii) $d\left(L, L^{\prime}\right) \leq D_{0}$, (iii) $\pi\left(L^{\prime}\right)=c$ and (iv) there exists an arc $\alpha$ connecting $L$ and $L^{\prime}$ whose projection to $R$ has no intersection with $c$ except at the end points. Then the number of $\gamma$-equivalence classes of axes in $S$ is dominated by

$$
-\frac{l}{\log \left(\tanh \left(D_{0} / 2\right)\right)} \text {. }
$$


Proof. We may assume that $L=\{i y \mid y>0\}$. We take $\theta_{0}$ $\left(0<\theta_{0}<\pi / 2\right)$ so that $\cosh D_{0}=\left(\cos \theta_{0}\right)^{-1}$ and set $\theta=\pi / 2-\theta_{0}$. Furthermore, we set

$$
T_{+}=\left\{r e^{i \theta} \mid 1 \leq r<e^{l}\right\} \quad \text { and } \quad T_{-}=\left\{r e^{i(\pi-\theta)} \mid 1 \leq r<e^{l}\right\} .
$$

Then $d\left(L, T_{+}\right)=D_{0}$ and $d\left(L, T_{-}\right)=D_{0}$. To estimate the number of $\gamma$-equivalence classes of elements in $S$, we have only to consider the maximal number $n$ of disjoint axes $L^{\prime}$ that are tangent to $T_{+}$or $T_{-}$.

Let $C$ be the Euclidean circle on $\mathbb{C}$ that is tangent to the segment $T_{+}$and has center $a>0$ with radius $r$. Then $r=a \sin \theta$, and the circle $C$ passes through two points,

$$
x_{1}=(1-\sin \theta) a \quad \text { and } \quad x_{2}=(1+\sin \theta) a .
$$

The ratio of these points is given by

$$
s=\frac{x_{2}}{x_{1}}=\frac{1+\sin \theta}{1-\sin \theta}=\frac{1+\cos \theta_{0}}{1-\cos \theta_{0}}=\frac{\cosh D_{0}+1}{\cosh D_{0}-1}=\frac{1}{\left(\tanh \left(D_{0} / 2\right)\right)^{2}} .
$$

Hence it is easy to see that

$$
n \leq 2 \cdot \frac{l}{\log s}=-\frac{l}{\log \left(\tanh \left(D_{0} / 2\right)\right)} .
$$

The following proposition gives a relationship between the hyperbolic distance of two axes and that of their images under a quasiconformal map.

Proposition 3.3 ([1]). Let $f$ be a $K$-quasiconformal automorphism of $\mathbb{H}$. Then there exists a constant $C=C(K)>0$ depending only on $K$ such that, for any two geodesics $L_{1}$ and $L_{2}$ in $\mathbb{H}$, the inequality

$$
K^{-1} \cdot d\left(L_{1}, L_{2}\right)-C \leq d\left(f\left(L_{1}\right)_{*}, f\left(L_{2}\right)_{*}\right) \leq K \cdot d\left(L_{1}, L_{2}\right)+C
$$

holds. The constant $C(K)$ satisfies $C(K) \rightarrow 0$ as $K \rightarrow 1$, and may be taken to be

$$
(1 / 2) \operatorname{arccosh}\left(2^{-(K-1)^{2}} e^{6(K+1)^{2} \sqrt{K-1}}\right) .
$$

The following proposition gives a sufficient condition for the maximal dilatations of quasiconformal maps to be bounded away from one.

Proposition $3.4([4])$. Let $R=\mathbb{H} / \Gamma$ be a Riemann surface. Suppose that $R$ satisfies the lower bound condition for a constant $\epsilon>0$ as well as the upper bound condition for a constant $M>0$ and a connected component $R_{M}^{*}$ of $R_{M}$. Let $B>0$ and $l>0$ be constants. Then 
there exists a constant $A_{0}=A_{0}(\epsilon, M, B, l)>1$ depending only on $\epsilon$, $M, B, l$ and satisfying the following conditions: Given a quasiconformal automorphism $f$ of $R$, suppose that there exist three disjoint axes $L_{i}(i=1,2,3)$ of hyperbolic elements of $\Gamma$ such that

(1) their projections $\pi\left(L_{i}\right)$ on $R$ are simple closed geodesics $c_{i}(i=$ $1,2,3)$ with $c_{i} \subset R_{M}^{*}$ and $l\left(c_{i}\right) \leq l$,

(2) $d\left(L_{1}, L_{2}\right) \leq B$

(3) $\tilde{f}\left(L_{1}\right)_{*}=L_{1}, \quad \tilde{f}\left(L_{2}\right)_{*}=L_{2}, \quad \tilde{f}\left(L_{3}\right)_{*} \neq L_{3}$ for a lift $\tilde{f}$ of $f$ to $\mathbb{H}$.

Then $K(f) \geq A_{0}$.

We now prove our theorems.

Proof of Theorem 2.1. We set $B:=D=D(M, l)$ in Proposition 3.4 and let $A_{0}=A_{0}(\epsilon, M, l)>1$ be a constant depending only on $\epsilon, M$ and $l$ obtained in Proposition 3.4. Setting $A=\min \left\{A_{0}, 2\right\}$, we prove the statement for $K_{0}=A^{1 /\left(N_{0}+1\right)}$. Namely, we show that, if $K(f)<K_{0}$, then there exists an integer $n \leq N_{0}$ such that $f^{n}$ is homotopic to the identity.

Let $\Gamma$ be a Fuchsian model of $R$. Furthermore let $L_{1}$ be an axis such that $\pi\left(L_{1}\right)=c$ and $\gamma_{1}$ the primitive hyperbolic element of $\Gamma$ with axis $L_{1}$. By applying Proposition 3.1 to $L_{1}$, we see that there exists an axis $L_{2}$ of a hyperbolic element $\gamma_{2}$ of $\Gamma$ such that $L_{1} \cap L_{2}=\emptyset$, $d\left(L_{1}, L_{2}\right) \leq D$ and $\pi\left(L_{1}\right)=\pi\left(L_{2}\right)$.

Let $\overline{\tilde{f}}$ be a lift of $f$ to $\mathbb{H}$ satisfying $\tilde{f}\left(L_{1}\right)_{*}=L_{1}$. Since $K(f)<$ $K_{0}=A^{1 /\left(N_{0}+1\right)}$, we have $K\left(f^{k}\right)<A$ for $k \leq N_{0}+1$. Then, by Proposition 3.3,

$$
\begin{aligned}
d\left(L_{1}, \tilde{f}^{k}\left(L_{2}\right)_{*}\right) & =d\left(\tilde{f}^{k}\left(L_{1}\right)_{*}, \tilde{f}^{k}\left(L_{2}\right)_{*}\right) \leq A \cdot d\left(L_{1}, L_{2}\right)+C(A) \\
& \leq 2 D+C(2)=2 D+(1 / 2) \operatorname{arccosh}\left(e^{54} / 2\right) \\
& \leq 2 D+27
\end{aligned}
$$

for all $k \leq N_{0}+1$.

We consider the set $S_{0}$ of all axes $L^{\prime}$ of hyperbolic elements of $\Gamma$ satisfying the following conditions: (i) $L_{1} \cap L^{\prime}=\emptyset$, (ii) $d\left(L_{1}, L^{\prime}\right) \leq$ $2 D+27$, (iii) $\pi\left(L^{\prime}\right)=c$ and (iv) there exists an arc $\alpha$ connecting $L_{1}$ and $L^{\prime}$ such that the projection of $\alpha$ to $R$ has no intersection with $c$ except at the end points. We see that the set $S^{\prime}=\left\{\tilde{f}^{k}\left(L_{2}\right)_{*}\right\}_{k=1}^{N_{0}+1}$ is contained in $S_{0}$. Indeed, by the proof of Proposition 3.1, the axis $L_{2}$ satisfies the property (iv), and since $\tilde{f}^{k}$ is a homeomorphism, the axes $\tilde{f}^{k}\left(L_{2}\right)_{*}$ satisfy the same property. The other properties (i), (ii), (iii) are also satisfied. 
By Proposition 3.2, the number of $\gamma_{1}$-equivalence classes of elements in $S_{0}$ is dominated by $N_{0}$. Hence there exist at least two elements in $S^{\prime}$, say $\tilde{f}^{m_{1}}\left(L_{2}\right)_{*}$ and $\tilde{f}^{m_{2}}\left(L_{2}\right)_{*}\left(1 \leq m_{1}<m_{2} \leq N_{0}+1\right)$, that are $\gamma_{1}$-equivalent to each other. Thus there exists $j \in \mathbb{Z}$ such that $\gamma_{1}^{j} \circ \tilde{f}^{n}\left(L_{2}\right)_{*}=L_{2}$, where $n=m_{2}-m_{1}\left(\leq N_{0}\right)$. With this $n$, we will prove that $f^{n}$ is homotopic to the identity. We set $F=\gamma_{1}^{j} \circ \tilde{f}^{n}$, which is a lift of $f^{n}$ to $\mathbb{H}$.

Suppose to the contrary that $f^{n}$ is not homotopic to the identity. We set $\chi(\gamma)=F \circ \gamma \circ F^{-1}$ for $\gamma \in \Gamma$. Then there exists $\gamma_{3} \in \Gamma$ such that $\chi\left(\gamma_{3}\right) \neq \gamma_{3}$. Setting $\gamma_{i}^{\prime}=\gamma_{3} \circ \gamma_{i} \circ \gamma_{3}^{-1}$ for $i=1,2$, we claim that either $\chi\left(\gamma_{1}^{\prime}\right) \neq \gamma_{1}^{\prime}$ or $\chi\left(\gamma_{2}^{\prime}\right) \neq \gamma_{2}^{\prime}$ is satisfied. Suppose that both $\chi\left(\gamma_{1}^{\prime}\right)=\gamma_{1}^{\prime}$ and $\chi\left(\gamma_{2}^{\prime}\right)=\gamma_{2}^{\prime}$ are satisfied. Since $\chi\left(\gamma_{i}\right)=\gamma_{i}$, we have $\beta \circ \gamma_{i} \circ \beta^{-1}=\gamma_{i}(i=1,2)$, where $\beta=\gamma_{3}^{-1} \circ \chi\left(\gamma_{3}\right)$. Thus, $\beta$ fixes all fixed points of $\gamma_{1}$ and $\gamma_{2}$. Since $\gamma_{1}$ and $\gamma_{2}$ are non-commutative, the Möbius transformation $\beta$ fixes four points and must be the identity. This contradicts that $\chi\left(\gamma_{3}\right) \neq \gamma_{3}$.

Hence either $F\left(\gamma_{3}\left(L_{1}\right)\right)_{*} \neq \gamma_{3}\left(L_{1}\right)$ or $F\left(\gamma_{3}\left(L_{2}\right)\right)_{*} \neq \gamma_{3}\left(L_{2}\right)$ is satisfied, and we may assume without loss of generality that $F\left(\gamma_{3}\left(L_{1}\right)\right)_{*} \neq$ $\gamma_{3}\left(L_{1}\right)$. Since $\pi\left(\gamma_{3}\left(L_{1}\right)\right)=\pi\left(L_{1}\right)=c$, we can apply Proposition 3.4 to the lift $F$ of $f^{n}$ and to the three axes $L_{1}, L_{2}$ and $\gamma_{3}\left(L_{1}\right)$. Then we have $K\left(f^{n}\right) \geq A_{0}$, a contradiction, since we assumed $K\left(f^{n}\right)<A \leq A_{0}$. Hence if $K(f)<A^{1 /\left(N_{0}+1\right)}$, then $f^{n}$ is homotopic to the identity.

Proof of Theorem 2.2. In the proof of Theorem 2.1, we can replace the inequality (1) with

$$
d\left(L_{1}, \tilde{f}^{k}\left(L_{2}\right)_{*}\right)=d\left(\tilde{f}^{k}\left(L_{1}\right)_{*}, \tilde{f}^{k}\left(L_{2}\right)_{*}\right)=d\left(L_{1}, L_{2}\right)=D .
$$

Hence we have only to replace the constant $2 D+27$ with $D$ in Theorem 2.1 .

\section{REFERENCES}

[1] A. Basmajian, Quasiconformal mappings and geodesic in the hyperbolic plane, Contemp. Math. 256 (2000), 1-4.

[2] A. F. Beardon, The Geometry of Discrete Groups, Grad. Texts in Math. 91, Springer, 1983.

[3] E. Fujikawa, The order of conformal automorphisms of Riemann surfaces of infinite type, Kodai Math. J. 26 (2003), 16-25.

[4] E. Fujikawa, Modular groups acting on infinite dimensional Teichmüller spaces, In the tradition of Ahlfors-Bers, III: Proceedings of the 2001 Ahlfors-Bers Colloquium, Contemp. Math., to appear.

[5] E. Fujikawa, H. Shiga and M. Taniguchi, On the action of the mapping class group for Riemann surfaces of infinite type, J. Math. Soc. Japan, to appear. 
[6] A. Yamada, On Marden's universal constant of Fuchsian groups II, J. Analyse Math. 41 (1982), 234-248.

Research Institute for Mathematical Sciences

KYOTO UNIVERSITY

Куото, 606-8502

JAPAN

Current address: Department of mathematical and Computing Sciences

Tokyo Institute of Technology

Oh-okayama Meguro-ku Tokyo, 152-8552

Japan

E-mail address: ege.fujikawa@is.titech.ac.jp 